\title{
A FRAMEWORK FOR THE AUTOMATION OF GENERALIZED STABILITY THEORY*
}

\author{
P. E. FARRELL ${ }^{\dagger}$, C. J. COTTER ${ }^{\ddagger}$, AND S. W. FUNKE ${ }^{\S}$
}

\begin{abstract}
The traditional approach to investigating the stability of a physical system is to linearize the equations about a steady base solution, and to examine the eigenvalues of the linearized operator. Over the past several decades, it has been recognized that this approach only determines the asymptotic stability of the system, and neglects the possibility of transient perturbation growth arising due to the nonnormality of the system. This observation motivated the development of a more powerful generalized stability theory (GST), which focuses instead on the singular value decomposition (SVD) of the linearized propagator of the system. While GST has had significant successes in understanding the stability of phenomena in geophysical fluid dynamics, its more widespread applicability has been hampered by the fact that computing the SVD requires both the tangent linear operator and its adjoint: deriving the tangent linear and adjoint models is usually a considerable challenge, and manually embedding them inside an eigensolver is laborious. In this paper, we present a framework for the automation of generalized stability theory, which overcomes these difficulties. Given a compact high-level symbolic representation of a finite element discretization implemented in the FEniCS system, efficient $\mathrm{C}++$ code is automatically generated to assemble the forward, tangent linear, and adjoint models; these models are then used to calculate the optimally growing perturbations to the forward model, as well as their growth rates. By automating the stability computations, we hope to make these powerful tools a more routine part of computational analysis. The efficiency and generality of the framework are demonstrated, with applications drawn from geophysical fluid dynamics, phase separation, and quantum mechanics.
\end{abstract}

Key words. generalized stability theory, adjoint models, tangent linear models, algorithmic differentiation, code generation, finite elements, FEniCS

AMS subject classifications. 34D10, 34D15, 34D20, 35B20, 25B25, 35B30, 35B35, 74S05

DOI. $10.1137 / 120900745$

1. Introduction. The stability of a physical system is a classical problem of mechanics, with contributions from authors such as Lagrange, Dirichlet, and Lyapunov [35]. Stability investigates the response of the system to small perturbations applied to a particular initial condition: if for every $\epsilon$ there exists a $\delta$-neighborhood of initial conditions such that their solutions remain within the $\epsilon$-neighborhood, then the system is stable at that initial condition; otherwise, the system is unstable.

The traditional approach for investigating the stability of physical systems was given by Lyapunov [39]. The nonlinear equations of motion are linearized about a base solution, and the eigenvalues of the linearized system are computed. If all eigenvalues have negative real part, then there exists a finite region of stability around

* Submitted to the journal's Software and High-Performance Computing section November 30, 2012; accepted for publication (in revised form) October 2, 2013; published electronically January 21, 2014.

http://www.siam.org/journals/sisc/36-1/90074.html

$\dagger$ Mathematical Institute, University of Oxford, Andrew Wiles Building, Radcliffe Observatory Quarter, Oxford OX2 6GG, UK (patrick.farrell@maths.ox.ac.uk). This author's work was supported by EPSRC grants EP/I00405X/1 and EP/K030930/1 and a Center of Excellence grant from the Norwegian Research Council to the Center for Biomedical Computing at Simula Research Laboratory.

${ }^{\ddagger}$ Department of Mathematics, Imperial College London, London, UK (colin.cotter@imperial. ac.uk).

${ }^{\S}$ Center for Biomedical Computing, Simula Research Laboratory, Oslo, Norway, and Applied Modelling and Computation Group, Department of Earth Science and Engineering, Imperial College London, London, UK (s.funke09@imperial.ac.uk). This author's work was supported by EP/I00405X/1, the Grantham Institute for Climate Change, and Fujitsu Laboratories of Europe Ltd. 
the initial condition: perturbations within that region decay to zero, and the system is asymptotically stable [51].

While this approach has had many successes, several authors have noted that it does not give a complete description of the finite-time stability of a physical system. While the eigendecomposition determines the asymptotic stability of the linearized equations as $t \rightarrow \infty$, some systems permit transient perturbations which grow in magnitude, before being predicted to decay. However, if the perturbations grow too large, the linearized approximation may no longer be valid, and the system may become unstable due to nonlinear effects. More specifically, this transient growth occurs when the system is nonnormal, i.e., when the eigenfunctions of the system do not form an orthogonal basis [55]. For example, Trefethen et al. [60] describe how the traditional approach fails to give accurate stability predictions for several classical problems in fluid mechanics; the problem is resolved by analyzing the nonnormality of the system in terms of pseudospectra [59].

Therefore, this motivates the development of a finite-time theory of stability to investigate and predict the transient growth of perturbations. While Lorenz [38] discussed the core ideas (without using modern nomenclature), the development of this so-called generalized stability theory (GST) has been driven by the work of B. F. Farrell and coworkers (e.g., $[16,17,18,19])$. The main idea is to consider the linearized propagator of the system, which is the operator (linearized about the time-dependent trajectory) that maps perturbations in the initial conditions to perturbations in the final state. Essentially, the propagator is the inverse of the tangent linear system associated with the nonlinear forward model, along with operators to set the initial perturbation and select the final perturbation. The perturbations that grow maximally over the time window are given by the singular functions of the propagator associated with the largest singular values. Since the linearized propagator depends on the base solution, it follows that the predictability of the system depends on the conditions of the base solution itself: some states are inherently more predictable than others $[38,30]$. This idea has made a significant impact in the meteorological and oceanographic communities, and has been used to investigate many aspects of geophysical fluid dynamics $[38,16,17,50,46,68,69]$. In the fluid dynamics community, this technique is occasionally referred to as direct optimal growth analysis [6].

While there are some applications of GST in other fields (e.g., [13, 41]), a large number of the applications of this powerful idea have been in the area of geophysical fluid dynamics. One reason for this is that the technique was invented in the meteorological community. Another reason is that nonnormality is important in such flows, whereas traditional eigenvalue analysis is sufficient for the normal case. A final reason is that the necessary adjoint and tangent linear models are commonly available in geophysical fluid dynamics, as they are necessary components for variational data assimilation, whereas the difficulty of implementing them inhibits the rapid application of GST in other scientific areas. Naumann [47] describes the automatic derivation of efficient adjoint and tangent linear models as "one of the great open challenges of High-Performance Scientific Computing."

The main contribution of this work is a system for automating the calculations required to perform a generalized stability analysis. Given a high-level description of a finite element discretization of the original time-dependent nonlinear model in the FEniCS framework [36], a representation of the tangent linear and adjoint models in the same high-level format are automatically derived at runtime [21]. These representations are then passed to a finite element form compiler [32], which emits 
efficient $\mathrm{C}++$ code for the assembly of the nonlinear forward model, the tangent linear model, and its adjoint [37]. The tangent linear and adjoint models are then used automatically in a robust implementation of the Krylov-Schur algorithm [27] for computing a partial singular value decomposition (SVD) of the model propagator. By automating the difficult steps of deriving the tangent linear model and its adjoint, GST becomes much more accessible: the analyst need only compactly describe a finite element discretization of the problem of interest, and then can simply request the fastest-growing perturbations and growth rates. The framework presented here is freely available under an open-source license as part of the dolfin-adjoint package (http://dolfin-adjoint.org).

This paper is organized as follows. Section 2 gives a brief overview of GST and mentions some applications in the literature. Section 3 describes the main contribution of this paper: how the calculations involved in GST can be entirely automated. This relies on the automatic derivation of tangent linear and adjoint models, as described in section 3.1. Finally, several examples are presented in section 4. The examples are drawn from several areas of computational science to emphasize the widespread applicability of the framework.

\section{Generalized stability theory.}

2.1. The SVD of the propagator. This presentation of GST theory will consider the stability of the system to perturbations in the initial conditions, but the same approach can be applied to analyzing the stability of the system to perturbations in other parameters.

Let $T$ be the time horizon of interest. Consider the solution of the model at the time $u_{T}$ as a pure function of the initial condition $u_{0}$ :

$$
u_{T}=M\left(u_{0}\right),
$$

where $M$ is the nonlinear propagator that advances the solution in time over the given finite-time window $[0, T]$. Other parameters necessary for the solution (e.g., boundary conditions, material parameters, etc.) are considered fixed. Assuming the model is sufficiently differentiable, the response of the model $M$ to a perturbation $\delta u_{0}$ in $u_{0}$ is given by

$$
\delta u_{T}=M\left(u_{0}+\delta u_{0}\right)-M\left(u_{0}\right)=\frac{\mathrm{d} M}{\mathrm{~d} u_{0}} \delta u_{0}+\mathcal{O}\left(\left\|\delta u_{0}\right\|^{2}\right) .
$$

Neglecting higher-order terms, the linearized perturbation to the final state is given by

$$
\delta u_{T} \approx \frac{\mathrm{d} M}{\mathrm{~d} u_{0}} \delta u_{0} \equiv L \delta u_{0},
$$

where $L$ is the linearized propagator (or just propagator) $\mathrm{d} M / \mathrm{d} u_{0}$ that advances perturbations in the initial conditions to perturbations to the final solution. For example, if $u_{T}$ is the solution of the linear ODE

$$
\frac{\mathrm{d} u}{\mathrm{~d} t}=A u
$$

then the propagator $L$ is given by

$$
L=e^{T A},
$$


where $e$ refers to the matrix exponential [44]. As discussed in [59, Figure 14.1], the initial behavior of $\left\|e^{T A}\right\|$ is governed by the numerical abscissa of $A$, while the asymptotic behavior is governed by the spectrum of $A$. Techniques such as generalized stability analysis and pseudospectral analysis are most useful for intermediate values of $T$, which are of interest for transient growth.

To quantify the stability of the system, we wish to identify perturbations $\delta u_{0}$ that grow the most over the time window $[0, T]$. For simplicity, equip both the initial condition and final solutions with the conventional inner product $\langle\cdot, \cdot\rangle$. We seek the initial perturbation $\delta u_{0}^{\star}$ of unit norm $\left\|\delta u_{0}^{\star}\right\|=\sqrt{\left\langle\delta u_{0}^{\star}, \delta u_{0}^{\star}\right\rangle}=1$ such that

$$
\delta u_{0}^{\star}=\underset{\left\|\delta u_{0}\right\|=1}{\arg \max }\left\langle\delta u_{T}, \delta u_{T}\right\rangle .
$$

Expanding $\delta u_{T}$ in terms of the propagator,

$$
\left\langle\delta u_{T}, \delta u_{T}\right\rangle=\left\langle L \delta u_{0}, L \delta u_{0}\right\rangle=\left\langle\delta u_{0}, L^{*} L \delta u_{0}\right\rangle,
$$

we see that the leading perturbation is the eigenfunction of $L^{*} L$ associated with the largest eigenvalue $\mu$, and the growth of the norm of the perturbation is given by $\sqrt{\mu}$. In other words, the leading initial perturbation $\delta u_{0}^{\star}$ is the leading right singular function of $L$, the resulting final perturbation $\delta u_{T}$ is the associated left singular function, and the growth rate of the perturbation is given by the associated singular value $\sigma$. The remaining singular functions offer a similar physical interpretation: if a singular function $v$ has an associated singular value $\sigma>1$, the perturbation will grow over the finite-time window $[0, T]$; if $\sigma<1$, the perturbation will decay over that time window. Note that the choice of $T$ is crucial: if $T$ is too large, the GST may predict contraction, even though significant transient growth may exist on a shorter timescale.

If the initial condition and final solution spaces are equipped with inner products $\langle\cdot, \cdot\rangle_{I} \equiv\left\langle\cdot, X_{I} \cdot\right\rangle$ and $\langle\cdot, \cdot\rangle_{F} \equiv\left\langle\cdot, X_{F} \cdot\right\rangle$, respectively, then the leading perturbations are given by the eigenfunctions

$$
X_{I}^{-1} L^{*} X_{F} L \delta u_{0}=\mu \delta u_{0}
$$

The operators $X_{I}$ and $X_{F}$ must be symmetric positive definite in order to define an inner product. In the finite element context, $X_{I}$ and $X_{F}$ are often the mass matrices associated with the input and output spaces, as these matrices induce the $L^{2}$ norm. All subsequent uses of the term SVD in this paper are taken to include this generalized SVD (2.8).

2.2. Computing the propagator. In general, the nonlinear propagator $M$ that maps initial conditions to final solutions is not available as an explicit function; instead, a partial differential equation (PDE) is solved. For clarity, let $m$ denote the data supplied for the initial condition. The PDE may be written in the abstract implicit form

$$
F(u, m)=0,
$$

with the understanding that $u_{0}=m$. We assume that for any initial condition $m$, the PDE (2.9) can be solved for the solution trajectory $u$; the nonlinear propagator $M$ can then be computed by returning the solution at the final time. Differentiating (2.9) with respect to the initial condition data $m$ yields

$$
\frac{\partial F}{\partial u} \frac{\mathrm{d} u}{\mathrm{~d} m}=-\frac{\partial F}{\partial m}
$$


the tangent linear system associated with the PDE (2.9). The term $\partial F / \partial u$ is the PDE operator linearized about the solution trajectory $u$ : therefore, it is linear, even when the original PDE is nonlinear. $\partial F / \partial m$ describes how the equations change as the initial condition data $m$ changes, and acts as the source term for the tangent linear system. $\mathrm{d} u / \mathrm{d} m$ is the prognostic variable of the tangent linear system (2.10) and describes how the solution changes with changes to $m$. To evaluate the action of the propagator $L$ on a given perturbation $\delta m$, the tangent linear system is solved with that particular perturbation and evaluated at the final time:

$$
L \delta m \equiv-\left.\left(\frac{\partial F}{\partial u}\right)^{-1} \frac{\partial F}{\partial m} \delta m\right|_{T} .
$$

Therefore, to automate the generalized stability analysis of a PDE (2.9), it is necessary to automatically derive and solve the associated tangent linear system (2.10). Furthermore, as discussed in section 3.2, all algorithms for computing the SVD of a matrix $A$ require its adjoint $A^{*}$; therefore, it is also necessary to automatically derive and solve the adjoint of the tangent linear system. If the PDE is linear and steady, then this derivation is straightforward; however, if the PDE is nonlinear and time dependent, the derivation of the associated tangent linear and adjoint systems is widely regarded as a major challenge, even with the assistance of algorithmic differentiation tools [47]. Another crucial concern is the efficiency of the derived models: the SVD computation requires many runs of the tangent linear and adjoint systems, and so their computational performance is of great importance if the stability analysis is to be tractable. However, by exploiting the special structure of finite element discretizations, it is possible to entirely automate the derivation of efficient tangent linear and adjoint models; this is the subject of the next section.

3. Automating generalized stability theory. The following sections explain in detail how the SVD computation is automated by combining the FEniCS framework [36], dolfin-adjoint, and SLEPc [27, 26].

3.1. Automating the generation of tangent linear and adjoint models. This section summarizes the novel approach taken for deriving the tangent linear and adjoint models associated with a given PDE solver. The main advantages over traditional approaches are its complete automation, its high performance, and its trivial parallelization. The approach is more fully described in [21].

The traditional approach to automatically deriving the tangent linear and adjoint models associated with a given PDE solver is to use algorithmic differentiation (AD, also known as automatic differentiation) tools [23, 47]. They primarily operate at the level of the source code (e.g., C++ or Fortran) that implements the discretization, having already developed the source code by hand. The main idea is to treat the model as a (very long) sequence of elementary instructions, such as additions and multiplications, each of which may be differentiated individually: the derived models are then composed using the chain rule applied forwards (in the tangent linear case) or backwards (in the adjoint case). This approach is sketched in Figure 3.1.

Naumann [47] states that "except for relatively simple cases, the differentiation of computer programs is not automatic despite the existence of many reasonably mature AD software packages." This approach treats the model at a very low level of abstraction, and many of the difficulties of AD stem from this fact.

A source-to-source AD tool operating on the low-level code must parse the source to build a representation of the sequence of elementary instructions as data. This pro- 


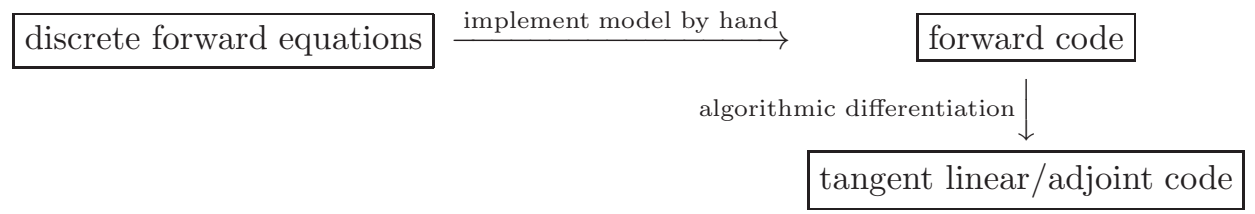

FIG. 3.1. The traditional approach to developing tangent linear and adjoint models. The forward model is implemented by hand, and its adjoint derived either by hand or (more often) with the assistance of an algorithmic differentiation tool.

cess is inherently fragile. The AD tool must handle complications such as preprocessor directives, parallel directives, libraries for which the source code is not immediately available, expressions with side effects, memory allocation, and aliasing. Correctly and efficiently handling these complications in generality is very difficult, which puts a significant burden on both tool developers and users of algorithmic differentiation.

However, with finite elements, it is possible to circumvent the problem of parsing source code. Finite element methods are based on a powerful high-level abstraction: the language of variational forms. This mathematical abstraction naturally allows for the discrete equations to be represented as data. In the FEniCS project [36], the discrete variational form is represented in the Unified Form Language (UFL) format, which is very similar to mathematical notation $[2,3]$. This representation is then passed to a specialized finite element form compiler [32], which emits optimized $\mathrm{C}++$ code to assemble the desired discrete equations. This approach has many advantages: it relieves the model developer of much of the manual labor (even complex models such as the Navier-Stokes can be written in tens of lines of code), the form compiler can employ specific optimizations that are complex to perform by hand [48], and the generated code can be tailored to the architecture at a very high level [42].

In the context of stability analysis, this approach has one other major advantage: by representing the equations to be solved as high-level data, the automated derivation of related models (such as the tangent linear and adjoint systems) becomes much more tractable. This high-level abstraction for the finite element model matches naturally with a higher-level abstraction for model differentiation: our approach takes the view that a model is a sequence of equation solves. This approach is implemented in the dolfin-adjoint software package [21]. Its strategy for deriving the tangent linear and adjoint models is now discussed.

When the dolfin-adjoint module is imported, all functions that solve equations or modify variable values are overloaded. In addition to providing their regular functionality, these overloaded functions build a tape of the forward model at runtime: the tape records all details of the forward evaluation necessary for evaluating the model at a different parameter. In low-level algorithmic differentiation, this consists of a record of all elementary operations performed, along with their arguments [23]; analogously, in the dolfin-adjoint case, the tape records all forward equations solved (in UFL format), their boundary conditions, their dependencies on previously computed values, etc. The tape contains a complete record of the discrete forward model and may be used to re-execute the forward model, which finds applications in PDE-constrained optimization and checkpointing.

The tape contains all information necessary to derive the tangent linear and adjoint models associated with the discrete forward model. For concreteness, consider 
the derivation of the tangent linear model. Each equation in the forward model induces an associated equation in the tangent linear model. Let $u_{k}$ be the variable solved for in equation $k$ of the forward model. Suppose the forward equation may be written as

$$
F_{k}\left(u_{k}, u_{k_{1}}, \ldots, u_{k_{N}}\right)=0
$$

where $F_{k}$ is a (possibly nonlinear) operator, and $u_{k_{1}}, \ldots, u_{k_{N}}$ are $N$ previously computed values on which the equation depends $\left(k_{i}<k \forall i\right)$. Let $\delta m$ be a perturbation to $m$ whose impact is to be quantified. By differentiating (3.1) with respect to $m$, we obtain the associated tangent linear equation

$$
\frac{\partial F_{k}}{\partial u_{k}} \dot{u}_{k}=\sum_{i=1}^{N}-\frac{\partial F_{k}}{\partial u_{k_{i}}} \dot{u}_{k_{i}}
$$

where

$$
\dot{u}_{j} \equiv \frac{\mathrm{d} u_{j}}{\mathrm{~d} m} \delta m
$$

is the tangent linear solution associated with $u_{j}$. If any boundary conditions are imposed strongly on (3.1), their homogenized counterparts are imposed strongly on (3.2); weakly imposed boundary conditions are handled naturally in the formulation. Note that $\dot{u}_{k_{1}}, \ldots, \dot{u}_{k_{N}}$ must be computed before the equation for $\dot{u}_{k}$ may be assembled, in the same way that $u_{k_{1}}, \ldots, u_{k_{N}}$ must be computed before the equation for $u_{k}$ may be assembled. Since (i) the tape represents $F_{k}$ symbolically in UFL format, (ii) the tape records which variables $u_{k_{1}}, \ldots, u_{k_{N}}$ equation $k$ depends on, and (iii) UFL supports the symbolic differentiation of operators with respect to their dependencies, the tangent linear equation (3.2) may be derived by symbolic manipulation of the data stored on the tape for the forward equation (3.1). Although the adjoint case is more complex, the associated adjoint equation may similarly be derived by symbolic manipulation of the tape; for full details, see [21].

By coupling the high-level representation of the forward model with this high-level differentiation approach, the tangent linear and adjoint versions of a model written in the FEniCS framework may be derived with almost no user intervention or effort [21]: this is because all of the necessary manipulation steps are fully automatable when the tape retains the symbolic structure of the equations. With the dolfin-adjoint software package, the discrete tangent linear and adjoint equations to be solved are symbolically derived in the exact same UFL format as the forward model, and passed to the same finite element compiler.

This alternative approach to automating the derivation of the tangent linear and adjoint models has several major advantages for generalized stability analysis. First, the derivation of the tangent linear and adjoint models is almost entirely automatic. In the example shown in section 3.3, the user need only add two lines of code: one to import the dolfin-adjoint library, and one to request the leading singular triplets. Second, the derived tangent linear and adjoint models approach optimal theoretical efficiency. This is crucial, as the SVD calculation requires many iterations of the tangent linear and adjoint models; the efficiency of the approach will be demonstrated in several examples in section 4 . Third, whereas applying algorithmic differentiation to a parallel code is a major research challenge [64, 22], this high-level approach parallelizes very naturally: if the forward model runs in parallel, the tangent linear and adjoint 
models will also [21]. In fact, there is no parallel-specific code in dolfin-adjoint-by operating on the discrete equations instead of the source code, the problem of parallelization dissolves. As the computational demands in problems of practical interest are usually very large, parallelization is a necessity if the GST framework is to be used in such cases.

3.2. Singular value decomposition. Once the propagator $L$ is available, its SVD may be computed. There are two main computational approaches. The first approach is to compute the eigendecomposition of the cross product matrix $L^{*} L$ (or $L L^{*}$, whichever is smaller). The second is to compute the eigendecomposition of the cyclic matrix

$$
H(L)=\left(\begin{array}{cc}
0 & L \\
L^{*} & 0
\end{array}\right) .
$$

The latter option is more accurate for computing the small singular values, but is more expensive [58]. As we are only interested in a small number of the largest singular triplets, the cross product approach is used throughout this work. Note that regardless of which approach is taken, the adjoint propagator $L^{*}$ is necessary to compute the SVD of $L$.

The algorithm used to compute the eigendecomposition of the cross product matrix is the Krylov-Schur algorithm [57], as implemented in SLEPc [27, 26]. As the cross product matrix is Hermitian, this algorithm reduces to the thick-restart variant [67] of the Lanczos method [34]. This algorithm was found experimentally to be faster than all other algorithms implemented in SLEPc for the computation of a small number of singular triplets, which is the case of interest in stability analysis.

Rather than computing and storing a dense matrix representation of the propagator, the action of the propagator is computed in a matrix-free fashion, using the tangent linear model. In turn, the entire time-dependent tangent linear model is not stored, but its action is implemented as the solution of several equations in sequence. In turn, the solution of each equation may optionally be achieved in a matrix-free fashion; the automatic derivation of the tangent linear and adjoint systems supports such an approach [21]. Similarly, the adjoint propagator is computed in a matrix-free fashion using the adjoint model. SLEPc elegantly supports such matrix-free computations through the use of PETSc shell matrices $[4,5]$.

3.3. Code example and implementation. In order to demonstrate the user interface of the proposed framework, a code example for a generalized stability analysis of the nonlinear Burgers' equation is given in Figure 3.2. The example is complete; nothing has been removed. Only two lines of code are added to the forward model to conduct the GST: one to import the dolfin-adjoint library, and one to perform the GST computation.

We now discuss the internals of the compute_gst function (Figure 3.3). The computation of the eigendecomposition is driven by SLEPc via the EPSSolve function. The main input to this routine is a PETSc shell matrix that represents the GST operator

$$
G=X_{I}^{-1} L^{*} X_{F} L .
$$

If no $X_{I}$ or $X_{F}$ are specified, the mass matrices of the associated function spaces are used by default. This shell matrix is equipped with a function that computes its 


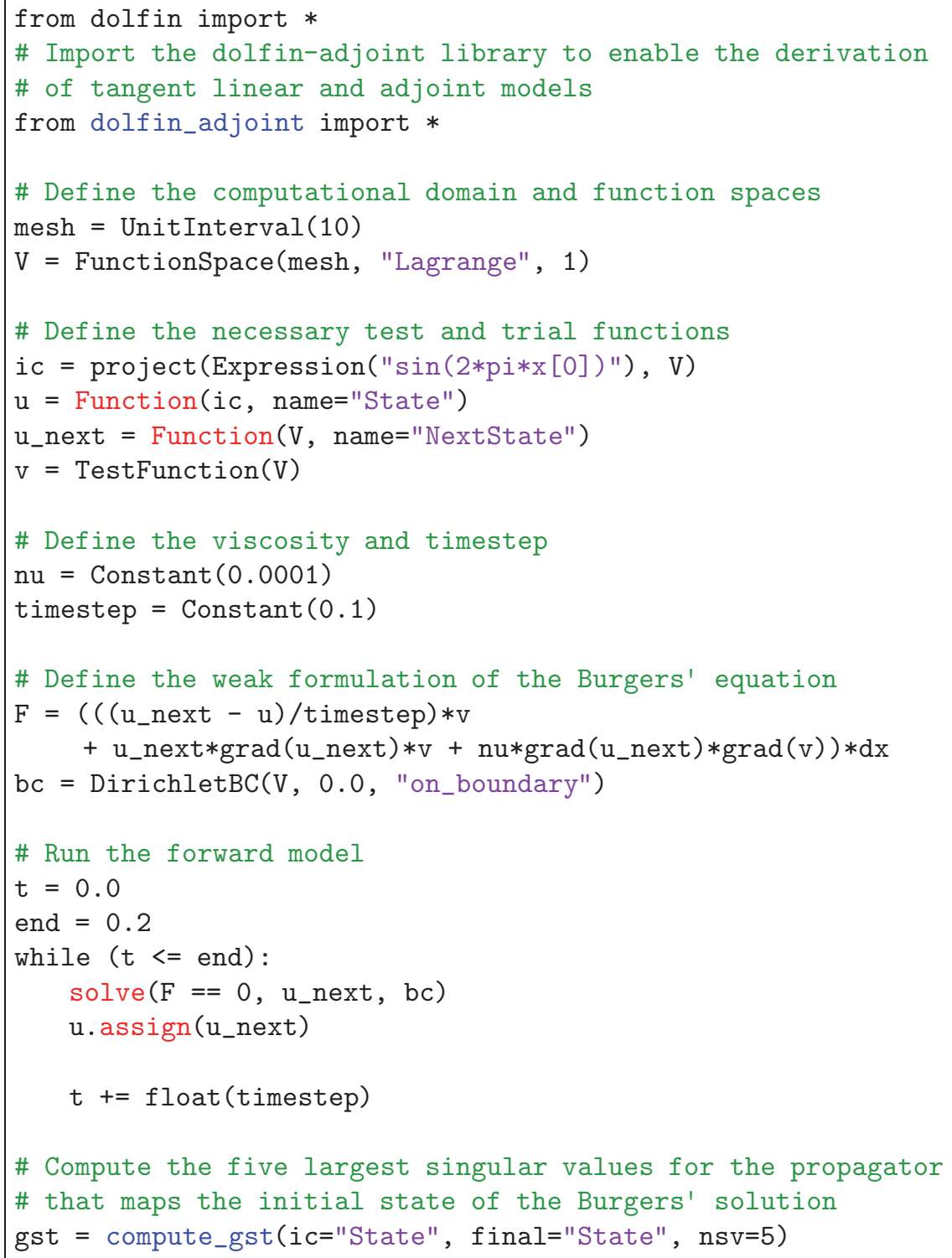

FIG. 3.2. The entire code to compute a generalized stability analysis of the nonlinear Burgers' equation. This code uses piecewise linear Lagrange finite elements for the spatial discretization (lines 8, 21-22), and implicit Euler for the temporal discretization (lines 21-22). The high-level approach leads to extremely compact and readable code. In order to use the framework presented here, only two additional lines are necessary (in blue): one to import the dolfin-adjoint library (line 4), and one to compute the SVD of the propagator associated with the forward model (line 36). The functions in red (lines 12-13, 29-30) are overloaded by dolfin-adjoint in order to record the information necessary for the derivation of the tangent linear and adjoint models as described in section 3.1. The compute_gst function (line 36) symbolically derives the tangent linear and adjoint models, creates a shell matrix to compute the action of the propagator, and embeds it inside a Krylov-Schur algorithm to compute the requested number of singular triplets. 


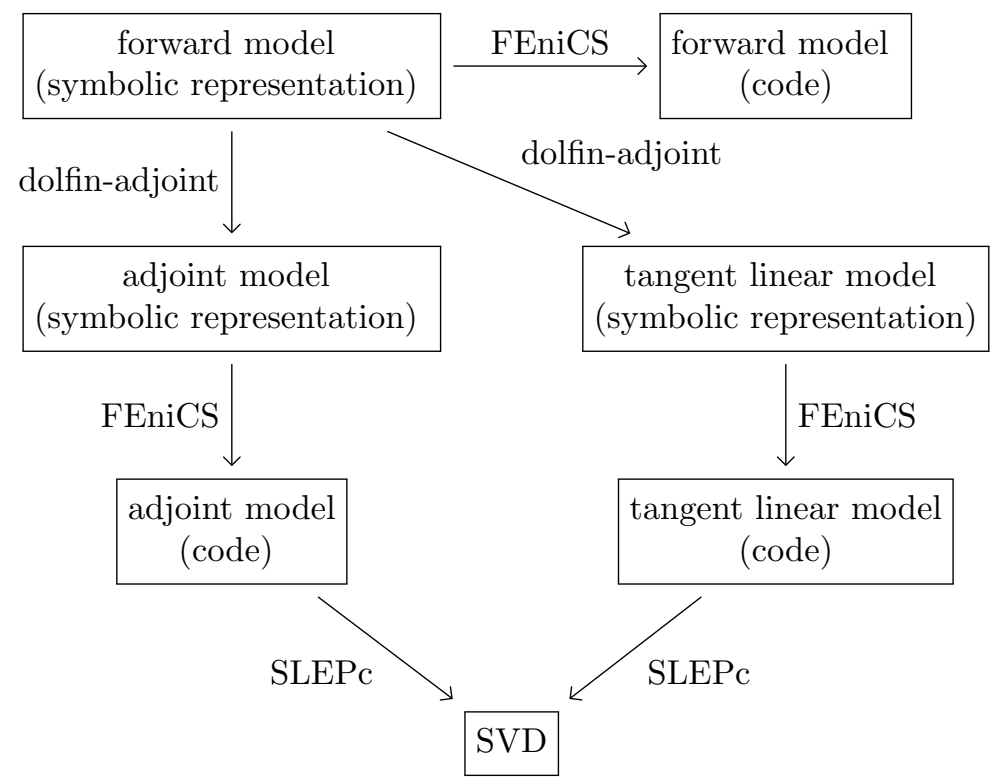

FIG. 3.3. The software components for computing the SVD. The user specifies the discrete forward equations in a high-level language similar to mathematical notation; the discrete forward equations are explicitly represented in memory in the UFL format. The in-memory representation of the associated tangent linear and adjoint systems is derived by dolfin-adjoint from the in-memory representation of the forward problem. Both the forward and adjoint equations are then passed to the FEniCS system, which automatically generates and executes the code necessary to compute the forward and adjoint solutions. Finally, SLEPc is used to compute the SVD.

action by composing the action of the four constituent matrices. The computation of the actions of $X_{F}$ and $X_{I}^{-1}$ is straightforward and is not discussed further.

The propagator $L$ is in turn represented as a shell matrix equipped with two operations, one for its action and one for its Hermitian action. The action of $L$ on a vector $\delta u$ is computed by inspecting the tape built by dolfin-adjoint during the initial forward run and deriving the tangent linear equation associated with each equation of the forward model, as discussed in section 3.1. The tape represents each forward equation symbolically in UFL format; the derived tangent linear equations are represented in the same UFL format, which means that efficient code for their assembly can be generated using the FEniCS system via automated code generation and just-in-time compilation. Each equation of the tangent linear system is solved in turn, with the source term $\delta u$ added to the right-hand side of the tangent linear equation associated with the forward variable that is defined to be the input of the propagator. Once the tangent linear equation associated with the output forward variable is solved, the tangent linear solution is returned as the action of the propagator.

The same strategy is used to compute the Hermitian action of the propagator, mutatis mutandis. The adjoint equations are solved in the opposite order to that of the forward model. Again, the assembly of the adjoint equations relies on the automated code generation technology of FEniCS. The perturbation on which $L^{*}$ is acting is added to the right-hand side of the adjoint equation associated with the output variable of the propagator, and the adjoint solution associated with the input variable of the propagator is returned. 
This implementation strategy has several advantages. SLEPc cleanly separates the algorithm for computing the eigendecomposition from the implementation of the matrices representing the propagator $L$ and the GST operator $G$. This means that developments in SLEPc (such as new algorithms, or improvements to existing ones) are immediately available. By exploiting the code generation facilities of FEniCS to implement the tangent linear and adjoint models, the implementation inherits all of its advantages, such as parallelism, efficiency, and generality. Finally, by relying on dolfin-adjoint for the automated derivation of the tangent linear and adjoint models, the user is relieved of the burden of manually deriving, implementing, and maintaining them.

In combination, this system allows for the flexible and efficient computation of generalized stability analyses, so long as the forward model is representable in the FEniCS system. The FEniCS system supports a wide variety of finite elements (including arbitrary order continuous and discontinuous Lagrange, Raviart-Thomas, Nédelec, Brezzi-Douglas-Marini, Crouzeix-Raviart [31]), distributed-memory unstructured meshes of complicated geometries, and any finite element discretization that can be represented in UFL. This includes sophisticated discretizations of complex PDEs, including Stokes with nonlinear rheology for mantle convection [65], viscoelastic deformation [54], the Landau-Lifshitz-Gilbert equation for micromagnetic simulations [1], and the coupled PDEs-ODEs describing the calcium release unit of sarcoplasmic reticulum in the heart [25].

4. Verification and applications. All applications are available under an opensource license as part of the dolfin-adjoint applications repository (http://dolfinadjoint.org). In all of the examples, the mass matrices of the input and output spaces were used to define the norms in (2.8). The benchmark tables show the minimum time of five experiments, performed on eight $2.13 \mathrm{GHz}$ Intel Xeon CPU cores with 12 GB memory.

4.1. Verification: The nonlinear Burgers' equation. The verification of the framework proceeds in two stages. First, the correctness of the tangent linear and adjoint models must be verified. Second, the correctness of the SVD must be verified.

The fundamental tool in verifying the correctness of the tangent linear and adjoint models is the Taylor remainder test. Suppose we have a black box for evaluating a function $f(x)$, and that we have a candidate function for its gradient $\nabla f$. The correctness of the gradient can be asserted by noting that by Taylor's theorem, the first order Taylor remainder

$$
|f(x+h \delta x)-f(x)| \rightarrow 0 \quad \text { at } O(h)
$$

converges to zero at first order, but that the Taylor remainder corrected with the gradient

$$
\left|f(x+h \delta x)-f(x)-h \delta x^{T} \nabla f\right| \rightarrow 0 \quad \text { at } O\left(h^{2}\right)
$$

converges to zero at second order. In this context, the function $f(u)$ is a functional of the solution $u$ of a PDE system $F(u, m)=0$ specified by parameters $m$, and its gradient $\nabla_{m} f(u(m))$ is computed in two different ways, once using the tangent linear model and once using its adjoint.

For the verification exercise, we choose as our model the nonlinear time-dependent Burgers' equation

$$
F(u, m) \equiv \frac{\partial u}{\partial t}+u \cdot \nabla u-\nu \nabla^{2} u=0
$$


TABLE 4.1

Verification of the tangent linear model. The Taylor remainders for the functional $\widehat{J}=J(u(m))$ are evaluated at a perturbed initial condition $\tilde{m} \equiv m_{0}+h \delta m$, where the perturbation direction $\delta m$ is pseudorandomly generated. As expected, the Taylor remainder incorporating gradient information computed using the tangent linear model converges at second order, indicating that the functional gradient computed using the tangent linear model is correct.

\begin{tabular}{ccccc}
\hline$h$ & $\left|\widehat{J}(\tilde{m})-\widehat{J}\left(m_{0}\right)\right|$ & Order & $\left|\widehat{J}(\tilde{m})-\widehat{J}\left(m_{0}\right)-\tilde{m}^{T} \nabla \widehat{J}\right|$ & Order \\
\hline $1 \times 10^{-3}$ & $1.8664 \times 10^{-5}$ & & $5.8991 \times 10^{-7}$ & \\
$5 \times 10^{-4}$ & $9.4796 \times 10^{-6}$ & 0.9773 & $1.4747 \times 10^{-7}$ & 2.000 \\
$2.5 \times 10^{-4}$ & $4.7766 \times 10^{-6}$ & 0.9888 & $3.6868 \times 10^{-8}$ & 2.000 \\
$1.25 \times 10^{-4}$ & $2.3975 \times 10^{-6}$ & 0.9944 & $9.2169 \times 10^{-9}$ & 2.000 \\
$6.25 \times 10^{-5}$ & $1.2010 \times 10^{-6}$ & 0.9972 & $2.3042 \times 10^{-9}$ & 2.000 \\
\hline
\end{tabular}

on some domain $\Omega \times[0, T]$, along with suitable boundary conditions and diffusivity coefficient $\nu$. The parameter $m$ is the initial condition for $u$. We choose our functional $J$ as

$$
J(u)=\int_{\Omega}\left|u_{T}\right|^{2} \mathrm{~d} x,
$$

the square of the $L^{2}$ norm of the solution evaluated at the end of time. By the chain rule, the gradient $\mathrm{d} J(u(m)) / \mathrm{d} m$ can be computed with

$$
\frac{\mathrm{d} J(u(m))}{\mathrm{d} m}=\left\langle\frac{\partial J}{\partial u}, \frac{\mathrm{d} u}{\mathrm{~d} m}\right\rangle,
$$

where $\mathrm{d} u / \mathrm{d} m$ is the solution of the associated tangent linear system (2.10). In this way, the automated derivation of the tangent linear system (2.10) from the nonlinear forward model (4.3) can be rigorously verified: the tangent linear solution is correct if and only if the second order Taylor remainder (4.2) converges at second order. In practice, computing the whole of the solution Jacobian $\mathrm{d} u / \mathrm{d} m$ is unnecessary, as we require only the action of the gradient $\mathrm{d} J / \mathrm{d} m$ on a particular perturbation $h \delta m$. In this case, it is sufficient to compute

$$
\left\langle\frac{\mathrm{d} J(u(m))}{\mathrm{d} m}, h \delta m\right\rangle=\left\langle\frac{\partial J}{\partial u}, h \frac{\mathrm{d} u}{\mathrm{~d} m} \delta m\right\rangle,
$$

where the action of the solution Jacobian $\mathrm{d} u / \mathrm{d} m$ on the perturbation $h \delta m$ is computed via

$$
\frac{\partial F}{\partial u}\left(h \frac{\mathrm{d} u}{\mathrm{~d} m} \delta m\right)=-h \frac{\partial F}{\partial m} \delta m .
$$

The Burgers' equation (4.3) is discretized in space using standard piecewise quadratic finite elements and discretized in time using the trapezoidal rule, and the resulting nonlinear system solved via Newton iteration. As described in section 3, the tangent linear model is automatically derived, with almost no user intervention. The results of the Taylor remainder test for the tangent linear model can be seen in Table 4.1. As expected, the Taylor remainders corrected with the functional gradient do indeed converge at second order, indicating that the computed gradient, the tangent linear solution, and the tangent linear equations are all correct. 
TABLE 4.2

Verification of the adjoint model. The Taylor remainders for the functional $\widehat{J}=J(u(m))$ are evaluated at a perturbed initial condition $\tilde{m} \equiv m_{0}+h \delta m$, where the perturbation direction $\delta m$ is pseudorandomly generated. As expected, the Taylor remainder incorporating gradient information computed using the adjoint model converges at second order, indicating that the functional gradient computed using the adjoint model is correct.

\begin{tabular}{ccccc}
\hline$h$ & $\left|\widehat{J}(\tilde{m})-\widehat{J}\left(m_{0}\right)\right|$ & Order & $\left|\widehat{J}(\tilde{m})-\widehat{J}\left(m_{0}\right)-\tilde{m}^{T} \nabla \widehat{J}\right|$ & Order \\
\hline $1 \times 10^{-3}$ & $4.0880 \times 10^{-5}$ & & $9.5164 \times 10^{-7}$ & \\
$5 \times 10^{-4}$ & $2.0678 \times 10^{-5}$ & 0.9833 & $2.3786 \times 10^{-7}$ & 2.000 \\
$2.5 \times 10^{-4}$ & $1.0398 \times 10^{-5}$ & 0.9917 & $5.9459 \times 10^{-8}$ & 2.000 \\
$1.25 \times 10^{-4}$ & $5.2141 \times 10^{-6}$ & 0.9958 & $1.4864 \times 10^{-9}$ & 2.000 \\
$6.25 \times 10^{-5}$ & $2.6107 \times 10^{-6}$ & 0.9979 & $3.7159 \times 10^{-9}$ & 2.000 \\
\hline
\end{tabular}

Similarly, the adjoint model may be verified by computing the gradient $\mathrm{d} J / \mathrm{d} m$ via the relation

$$
\frac{\mathrm{d} J(u(m))}{\mathrm{d} m}=-\left\langle\lambda, \frac{\partial F}{\partial m}\right\rangle,
$$

where $\lambda$ is the solution of the adjoint equation

$$
\left(\frac{\partial F}{\partial u}\right)^{*} \lambda=\frac{\partial J^{*}}{\partial u}
$$

The results of the Taylor remainder test for the adjoint model can be seen in Table 4.2. Again, the Taylor remainders corrected with the functional gradient do indeed converge at second order, indicating that the computed gradient, the adjoint solution, and the automatically derived adjoint equations are all correct.

With the correctness of the tangent linear and adjoint models established, the correctness of the SVD was verified. As described in section 3.2, in practical computations the propagator is never represented as a matrix: instead, its action is computed using the tangent linear model. However, for verification purposes, a dense matrix representation $U \Sigma V^{*}$ was computed by performing the full SVD of the propagator and multiplying the output matrices together. (This calculation was expensive and is unnecessary in the general case: the computation was performed merely for the purposes of verification.) The action of this dense matrix was compared against the matrix-free action with the tangent linear model on hundreds of random vectors $t$ (with each component drawn from $\mathcal{U}(0,1)$ ), by asserting that

$$
\left\|\left(U \Sigma V^{*}\right) t-L t\right\|<\epsilon
$$

for each $t$, with $\epsilon=10^{-7}$. Additionally, the matrix-free action of $L$ was computed on each right singular vector $v$, and the result compared to the prediction of the associated left singular vector from the SVD by asserting that

$$
\|u-L v\|<\epsilon
$$

for each $v$.

Finally, the relevance of the computed SVD was verified by running the nonlinear forward model with the initial condition perturbed with the leading right singular vector. The actual growth rate of the perturbation was compared with the growth rate predicted from the singular value; the prediction matched the actual growth rate to within $1 \%$. This confirms the physical utility of the SVD for predicting the dynamics of small perturbations to the initial condition. 


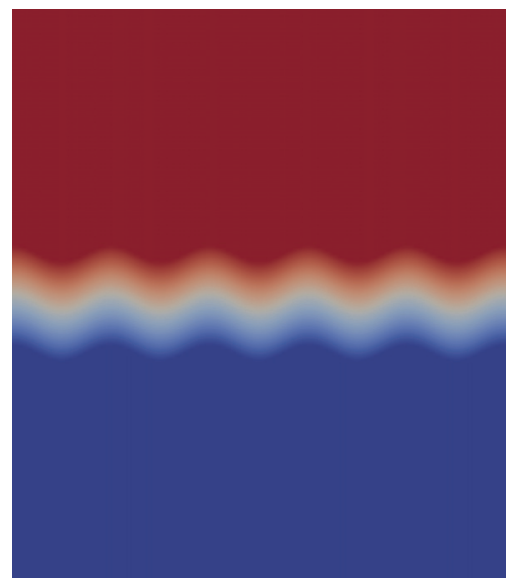

initial salinity

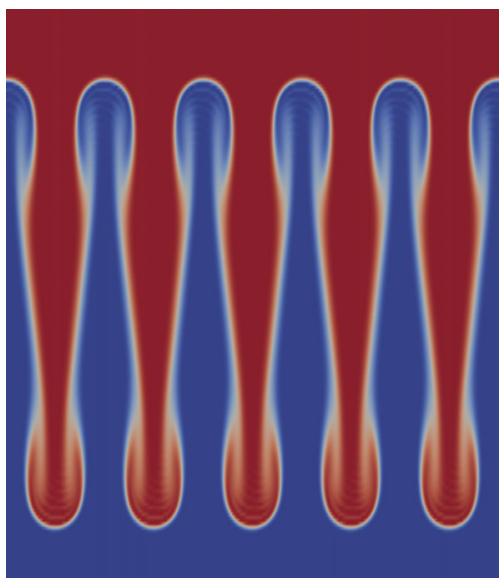

final salinity

FIG. 4.1. The phenomenon of salt fingering. Warm salty water overlies cold fresh water. If a parcel of warm salty water sinks downwards into the colder region, the heat of the parcel is diffused away much faster than its salt, thus making the parcel denser, and causing it to sink further. Left: the initial condition for salinity, using the perturbed interface of [49]. Right: the final salinity, at $T=0.05$.

4.2. Navier-Stokes: Double-diffusive salt fingering. In the ocean, the diffusivity coefficient of temperature is approximately two orders of magnitude larger than the diffusivity coefficient of salinity. Suppose warm salty water lies above colder, less salty water. If a parcel of warm salty water sinks downwards into the colder region, the heat of the parcel will diffuse away much faster than its salt, thus making the parcel denser, and causing it to sink further. Similarly, if a parcel of cold, less salty water rises into the warmer region, it will gain heat from its surroundings much faster than it will gain salinity, making the parcel more buoyant. This phenomenon is referred to as "salt fingering" [56] (Figure 4.1) and has been observed in many real-world oceanographic contexts [62]. An initial investigation of this phenomenon using the tools of GST was presented in [15].

Özgökmen and Esenkov [49] used a numerical model to investigate asymmetry in the growth of salt fingers caused by nonlinearities in the equation of state. In this work, we investigate the stability of the proposed configuration to small perturbations and examine what this means for its utility as a numerical benchmark. The twodimensional vorticity-streamfunction formulation of the Navier-Stokes equations is coupled to two advection equations for temperature and salinity:

$$
\begin{aligned}
\frac{\partial \zeta}{\partial t}+\nabla^{\perp} \psi \cdot \nabla \zeta & =\frac{\operatorname{Ra}}{\operatorname{Pr}}\left(\frac{\partial T}{\partial x}-\frac{1}{R_{\rho}^{0}} \frac{\partial S}{\partial x}\right)+\nabla^{2} \zeta, \\
\frac{\partial T}{\partial t}+\nabla^{\perp} \psi \cdot \nabla T & =\frac{1}{\operatorname{Pr}} \nabla^{2} T, \\
\frac{\partial S}{\partial t}+\nabla^{\perp} \psi \cdot \nabla S & =\frac{1}{\operatorname{Sc}} \nabla^{2} S, \\
\nabla^{2} \psi & =\zeta,
\end{aligned}
$$

where $\zeta$ is the vorticity, $\psi$ is the streamfunction, $T$ is the temperature, $S$ is the salinity, and $\mathrm{Ra}, \mathrm{Sc}, \mathrm{Pr}$, and $R_{\rho}^{0}$ are nondimensional parameters. Periodic boundary conditions are applied on the left and right boundaries; for full details of the remaining boundary 


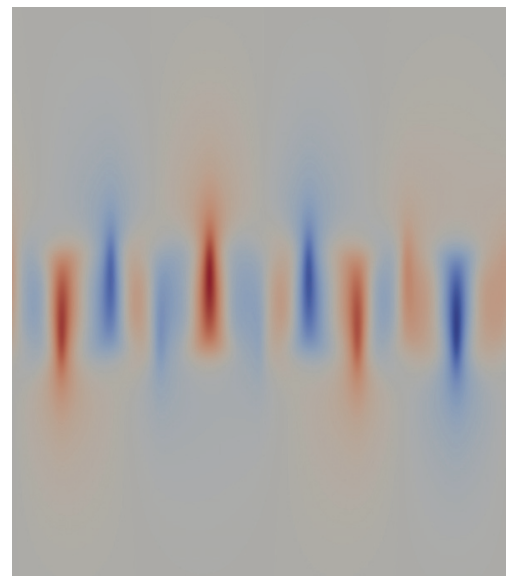

initial salinity perturbation

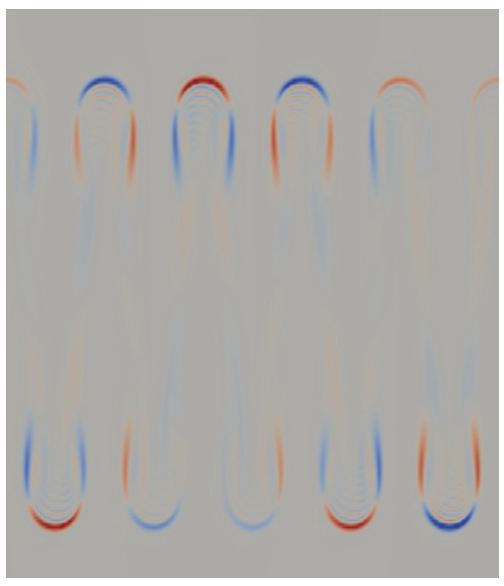

final salinity perturbation

FIG. 4.2. The leading perturbation to the salt fingering system. When the perturbation on the left is applied to the initial condition for salinity in the discretized model, the perturbation grows with a growth factor $\sigma \approx 2235$, resulting in a much larger perturbation to the final salinity.

conditions and values of the numerical parameters, see [49]. The configuration consists of two well-mixed layers (i.e., of homogeneous temperature and salinity) separated by an interface. To activate the instability, [49] added a sinusoidal perturbation to the initial salinity field (Figure 4.1).

To investigate the possibility of a secondary instability about this perturbed initial condition, the framework of GST was applied. The PDE was discretized in space using standard piecewise linear finite elements, and first order $\theta$-timestepping was employed in time with $\theta=0.6$. This value of $\theta$ was chosen to damp the over- and undershoots associated with the Galerkin advection of salinity [12, section 5]; an improved implementation would use a more sophisticated advection scheme. At each timestep, the entire discretized nonlinear system was solved with Newton iteration. The solution trajectory was computed using the initial sinusoidal perturbation to the salinity field, the propagator was linearized about that trajectory, and the leading ten singular triplets were computed. This calculation was repeated on several refinements of a structured mesh, up to $300 \times 300$ cells, and with timesteps ranging from $1 \times 10^{-3}$ to $1.25 \times 10^{-4}$.

The leading input perturbation is plotted in Figure 4.2, along with the resulting linear perturbation to the final state. As visible in the figure, the leading perturbation encourages the growth of some fingers, while retarding the growth of others. We identified a number of unstable modes which result in an uneven distribution of salt finger lengths; the physical mechanism is that longer fingers retard the growth of the shorter fingers since incompressibility requires a return flow in the opposite direction on either side of each finger. All ten perturbations computed were found to grow over the time interval $[0,0.05]$; the leading perturbation grew in norm by a factor of approximately 2235 over the time window. This secondary instability was first observed in [40], where these perturbations were activated by the use of unstructured meshes.

The performance was benchmarked by recording the runtimes of the forward, tangent linear, and adjoint models on a coarser configuration with a structured mesh of $50 \times 50$ cells and a timestep of $1 \times 10^{-3}$. The numerical results can be seen in 
TABLE 4.3

Timings for the salt fingering simulation for computing the perturbation that grows optimally to $T=0.05$. The optimal perturbation is obtained after 24 tangent linear and adjoint model solves. The table shows the runtime for the forward and the averaged timings for the tangent linear and adjoint solves. As can be seen, the tangent linear and the adjoint models take approximately $40 \%$ of the cost of the forward model. The optimal ratio is approximately 1.33.

\begin{tabular}{ccc}
\hline & Runtime (s) & Ratio \\
\hline Forward model & 165.89 & \\
Tangent linear model (averaged) & 65.25 & 1.39 \\
Adjoint model (averaged) & 68.71 & 1.41 \\
\hline
\end{tabular}

Table 4.3. During the forward solve, the Newton solver typically converges after three iterations. As both the adjoint and the tangent linear models replace each Newton solve with one linear solve, a coarse estimate of the optimal performance is that the tangent linear and adjoint models should take 33\% of the runtime of the forward model, for an optimal ratio of 1.33. (Efficiency results for derived models always include the cost of the forward model, as running the forward model is necessary to run derived models [47].) The numerical results yield a value of approximately $40 \%$ of the cost of the forward model; the tangent linear and the adjoint models approach optimal performance.

4.3. Cahn-Hilliard: Phase separation. The Cahn-Hilliard equation is a PDE which describes the process of phase separation, in which two components of a mixed binary fluid separate to form pure regions of each component [9]. The equation has also found applications in image processing for evolving object contours [10], and in astrophysics for modeling the evolution of Saturn's rings [61]. The Cahn-Hilliard equation is a nonlinear fourth order parabolic equation:

$$
\begin{aligned}
\frac{\partial c}{\partial t}-\nabla \cdot M\left(\nabla\left(\frac{\mathrm{d} f}{\mathrm{~d} c}-\lambda \nabla^{2} c\right)\right) & =0 & \text { on } \Omega, \\
M\left(\nabla\left(\frac{\mathrm{d} f}{\mathrm{~d} c}-\lambda \nabla^{2} c\right)\right) & =0 & \text { on } \partial \Omega, \\
M \lambda \nabla c \cdot n & =0 & \text { on } \partial \Omega,
\end{aligned}
$$

where $c$ is the prognostic concentration field ( $c=1$ is one fluid, $c=0$ the other), $f$ is the (prescribed) chemical potential, $n$ is the outward unit normal, and $\lambda$ and $M$ are scalar constants. In order to apply standard continuous finite elements, the fourth order equation is broken up into two coupled second order equations and a mixed P1-P1 finite element discretization applied [66].

Generalized stability analysis was employed to investigate the stability of the evolution of the Cahn-Hilliard system from a randomly perturbed initial condition on the domain $\Omega=[0,2]^{2}$. The initial condition was given by the one-dimensional profile

$$
c_{0}=c(t=0)=e^{-30(x-1)^{2}} .
$$

The constants were set to $\lambda=10^{-2}$ and $M=1$, and $f=100 c^{2}(1-c)^{2}$. The initial (at $t=0$ ) and final (at $t=5 \times 10^{-4}$ ) conditions for the simulation are presented in Figure 4.3. The mesh had 150 elements in both the $x$ - and $y$-directions, leading to a mixed function space with 90,602 degrees of freedom. The timestep $\Delta t$ was set to $5 \times 10^{-6}$. The simulations were run in parallel across eight cores using MPI. 


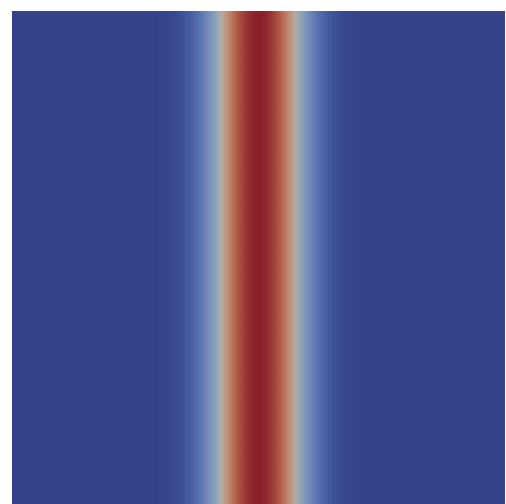

initial concentration

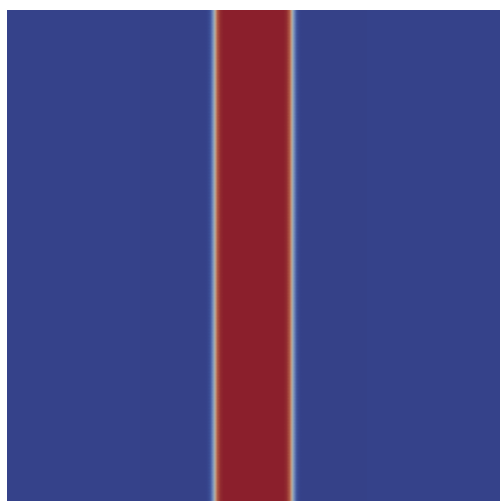

final concentration

FIG. 4.3. The initial and final conditions for the Cahn-Hilliard simulation. The color bar ranges from 0 to 1 .

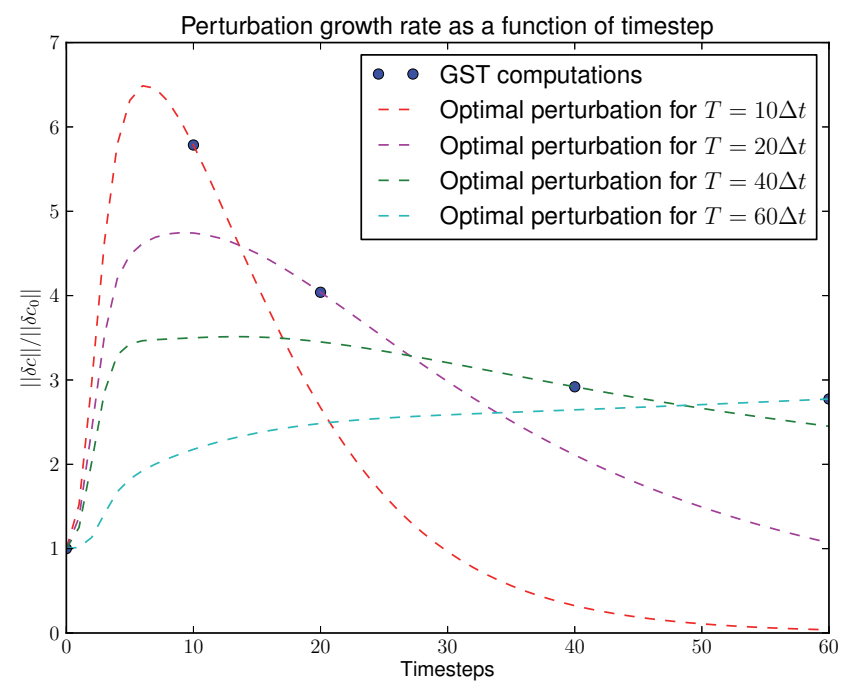

FIG. 4.4. The growth rate of the optimal perturbation computed using GST at various times (solid dots), and the growth rate of the optimal perturbation associated with various timesteps, computed using the nonlinear model (dashed lines). Note that the choice of $T$ is crucial. To compute the dashed curves, the identified perturbation was scaled to have norm $\left\|\delta c_{0}\right\|=10^{-7}$, and was added to the unperturbed initial condition. The nonlinear model was then executed with this perturbed initial condition and the results compared to the original unperturbed nonlinear trajectory. The fact that the dashed curves (observed from the nonlinear model) match the GST predictions indicates that the GST analysis is correct.

The generalized stability analysis was used to compute the optimally linearly growing perturbations to the initial condition for concentration and their growth rates at times $T=10 \Delta t, 20 \Delta t, 40 \Delta t$, and $60 \Delta t$. The optimal growth rates computed using GST for these values of $T$ are shown in Figure 4.4 (solid dots). In general, the perturbation that grows optimally to a time $T_{1}$ will be different from the perturbation that grows optimally to a time $T_{2} \neq T_{1}$; that is, the singular vectors are sensitive to 

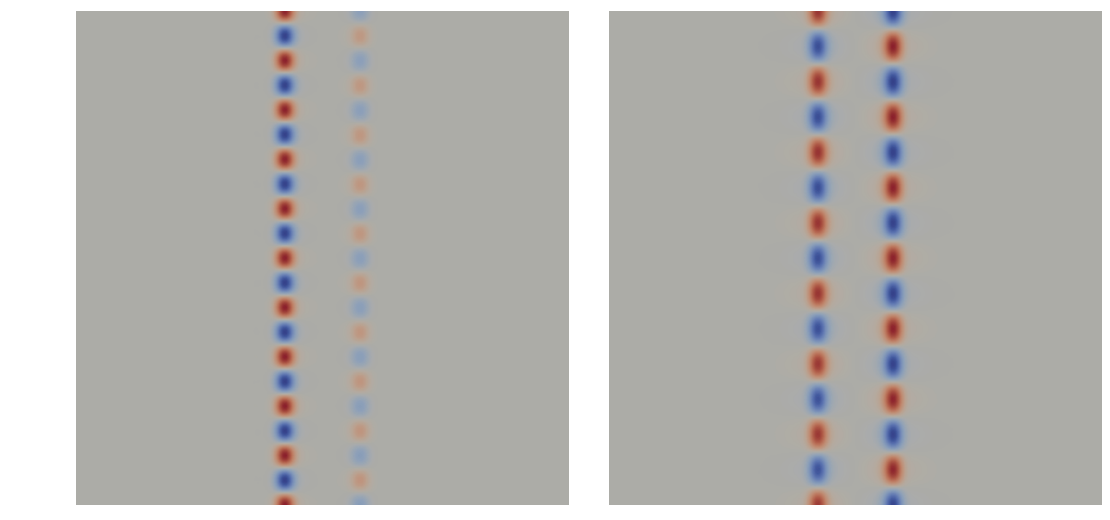

tb
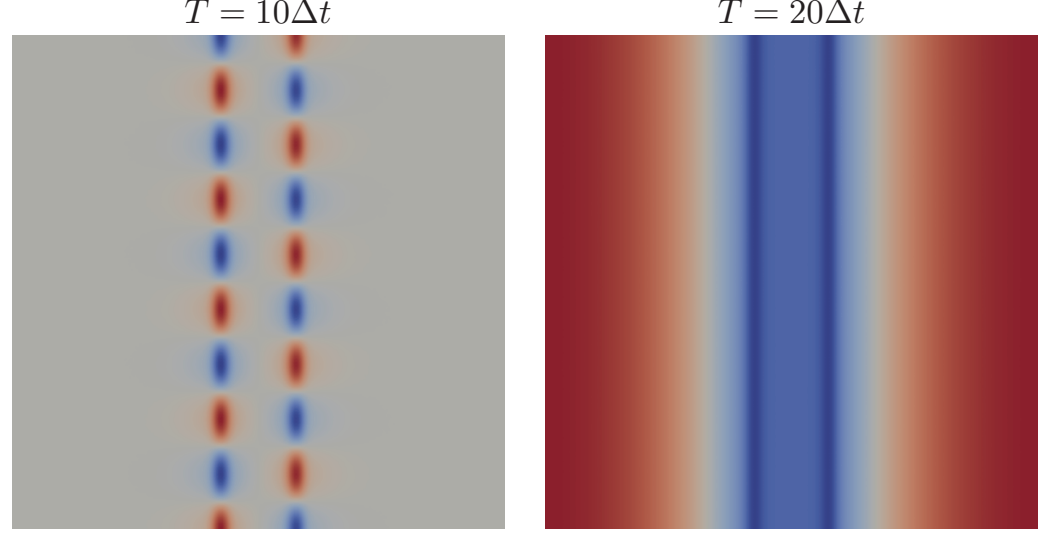

$T=40 \Delta t$

$T=60 \Delta t$

FIG. 4.5. The perturbation to the Cahn-Hilliard concentration that grows optimally (equivalently, the leading singular vector of the propagator), displayed for various integration periods. As the propagator is linear by definition, the scales of the perturbations do not matter, and so the perturbations are normalized to have unit norm. The optimal perturbation clearly depends on the time for which the propagator is defined.

the integration period of the propagator [30, p. 220]. This is indeed the case for the GST analysis of the Cahn-Hilliard system. The leading singular vectors of the propagator defined with respect to various times is shown in Figure 4.5.

To further verify the utility of GST, the nonlinear model was perturbed with each identified optimal perturbation in order to compare the growth rates predicted by the GST with the actual growth rates observed. The predictions and observations match closely, indicating that the GST is indeed predicting the quantitative behavior of the system (Figure 4.4, dashed lines). The growth curves of the perturbations demonstrate the phenomenon of transient growth: initial growth in magnitude over some finitetime horizon, followed by asymptotic decay. Such phenomena are characteristic of nonnormal systems [59].

The runtimes of the forward, tangent linear, and adjoint models for the setup with $T=10 \Delta t$ are shown in Table 4.4. For this configuration, the Newton solver typically converges after four iterations during the forward simulation. Therefore, the optimal performance can be estimated to be $25 \%$ of the runtime of the forward model, for an optimal ratio of 1.25 . The benchmark results yield a value of $27 \%$ of the cost of the forward model; the tangent linear and adjoint models approach optimal efficiency. 
TABLE 4.4

Timings for the Cahn-Hilliard simulation for computing the perturbation that grows optimally to $T=10 \Delta t$. The perturbation is obtained after 72 tangent linear and adjoint model solves. The table shows the runtime for the forward and the averaged timings for the tangent linear and adjoint solves. The optimal ratio is approximately 1.25 .

\begin{tabular}{ccc}
\hline & Runtime (s) & Ratio \\
\hline Forward model & 66.63 & \\
Tangent linear model (averaged) & 17.64 & 1.26 \\
Adjoint model (averaged) & 17.92 & 1.27 \\
\hline
\end{tabular}

4.4. Gross-Pitaevskii: Soliton solutions. The Gross-Pitaevskii equation $[24,52]$ is a nonlinear Schrödinger equation that describes the dynamics of a quantum system of identical bosons. The nondimensional equation governing the evolution of the wavefunction $\Psi$ is given by

$$
i \frac{\partial \Psi}{\partial t}+\nabla^{2} \Psi+s|\Psi|^{2} \Psi=0
$$

where $s$ is a parameter ( $s=1$ is the focusing case, $s=-1$ the defocusing case). In particular, the Gross-Pitaevskii equation describes the behavior of Bose-Einstein condensates, a state of matter observed when a dilute gas of bosons is cooled to temperatures close to absolute zero $[8,14]$. Bose-Einstein condensates are of considerable interest as they permit black hole analogues: systems from which acoustic perturbations, rather than light, are unable to escape [63]. This could potentially allow the laboratory-scale experimental investigation of the physics of black holes [20,33].

GST was employed to investigate the stability of the one-dimensional soliton solution of the focusing Gross-Pitaevskii equation

$$
\Psi=\sqrt{2} \frac{\exp \left(\frac{i}{2} x+\frac{3 i}{4} t\right)}{\cosh (x-t)}
$$

to perturbations in the initial condition. The Gross-Pitaevskii equation was solved with piecewise linear finite elements on the domain $\Omega=[-10,10]$ with periodic boundary conditions applied. The initial condition was achieved by pointwise evaluation of (4.21), and the equations were advanced in time from 0 to $T$ using the implicit midpoint rule. The interval was discretized with $N=480$ elements, and the timestep was set to $\Delta t=0.03125$.

The results of the GST calculation for various times are shown in Figure 4.6(a). In this example, approximately linear growth of the optimal perturbations is observed. For $T>10$, all GST calculations yielded very similar perturbations (Figure 4.6(b) shows the perturbation for $\mathrm{T}=50 \Delta t$ ).

This optimal perturbation corresponds to shifting along the family of soliton solutions parameterized by their amplitude. Since each member of this family has a different speed, perturbing in this direction leads to a similar-shaped soliton moving at a different speed, hence the linear growth in the perturbation. This indicates that the soliton solutions are stable. This is illustrated in Figure 4.7.

The timing results are given in Table 4.5. For this example, the model has only one spatial dimension, which makes the linear solves computationally cheap. As a consequence, the cost of the linear solves does not dominate the cost of the symbolic manipulation for low resolutions $(N=480)$, and so the efficiency ratio is suboptimal. However, as the mesh resolution is increased $(N=12,000)$, the cost of the linear 


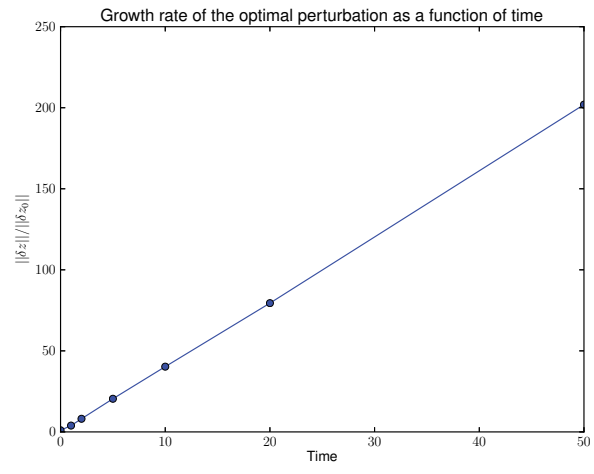

(a)

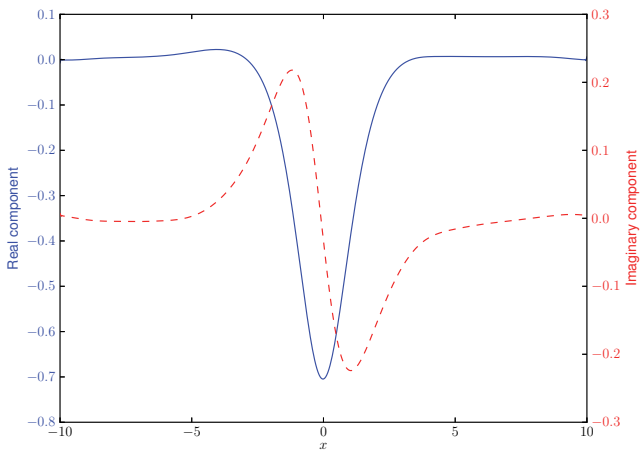

(b)

FIG. 4.6. (a) The growth rate of the optimal perturbation to Gross-Pitaevskii system as a function of time. The optimal perturbations associated with times $T>10$ are very similar. The linear growth of this perturbation was verified using the original nonlinear model up to $T=500$. (b) The optimal perturbation associated with time $T=50 \Delta t$. The solid line is the real component, while the dashed line is the imaginary component.

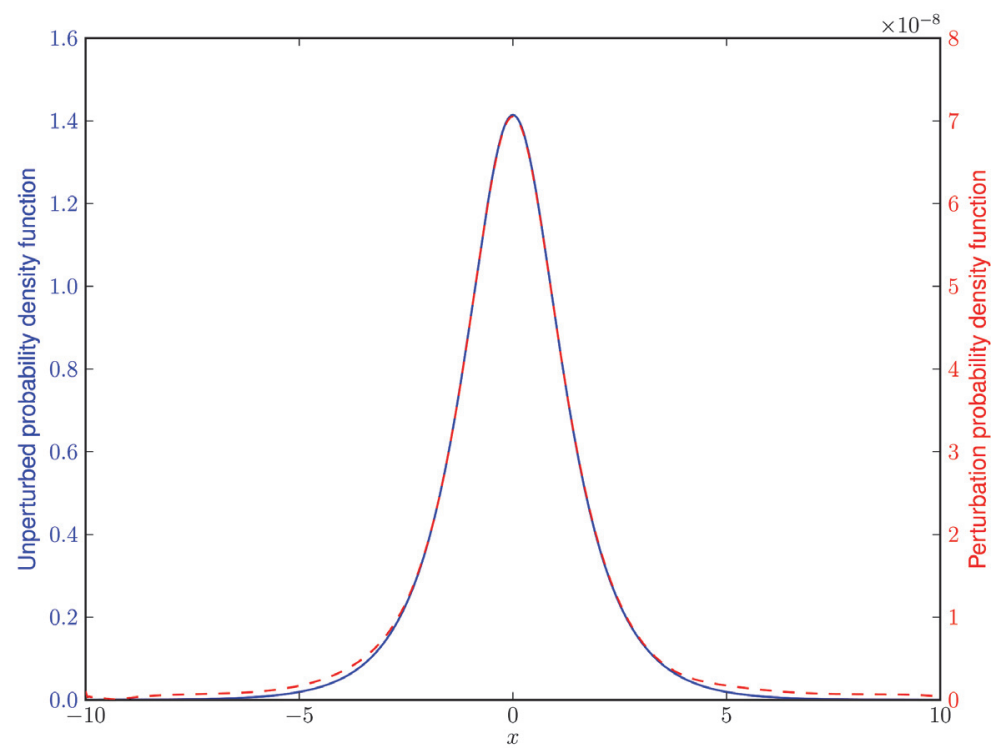

FIG. 4.7. The probability density functional for the unperturbed Gross-Pitaevskii soliton initial condition (solid line) and the optimal perturbation associated with times $T>10$ (dashed line). The perturbation corresponds to shifting to a higher (or lower, with negative coefficient) amplitude soliton solution; this is evident since the perturbation has almost the same shape as the soliton itself, but with slightly wider support. Higher (lower) amplitude soliton solutions have greater (lesser) speeds, and so the growth rate is linear in time.

solves increases, while the cost of the symbolic manipulation does not. Therefore, as the mesh is refined, the efficiency ratio approaches the optimal value. Of course, for one-dimensional problems, such fine discretizations are often unnecessary; however, the asymptotic regime is rapidly reached for problems of two or more dimensions, as in the previous examples. 
TABLE 4.5

Timings for the Gross-Pitaevskii simulation for computing the perturbation that grows optimally to $T=10$. The perturbation is obtained after 16 tangent linear (TLM) and adjoint model (ADM) solves. The table shows the runtime for the forward and the averaged timings for the tangent linear and adjoint solves. The Newton solver converges on average after two Newton iterations, which means that the optimal ratio is approximately 1.5. With low resolution $(N=480)$, the cost of the linear solves does not dominate the symbolic manipulation; as the mesh is refined $(N=12,000)$, the linear solves become the dominant cost, and the efficiency ratio approaches the optimal value.

\begin{tabular}{c|cc|cc|cc}
\hline Mesh elements & \multicolumn{2}{|c|}{$N=480$} & \multicolumn{2}{c|}{$N=6,000$} & \multicolumn{2}{c}{$N=12,000$} \\
& Runtime (s) & Ratio & Runtime (s) & Ratio & Runtime (s) & Ratio \\
\hline Forward model & 11.84 & & 58.06 & & 109.67 & \\
TLM (averaged) & 23.88 & 3.02 & 47.13 & 1.81 & 55.44 & 1.51 \\
ADM (averaged) & 24.50 & 3.07 & 51.63 & 1.89 & 58.88 & 1.54 \\
\hline
\end{tabular}

5. Conclusions. Generalized stability theory is a powerful tool for investigating the dynamics of physical systems, but the difficulty of implementing it has been a major impediment to its widespread application. The core contribution of this paper has been to remove this barrier. By employing a new high-level symbolic approach to automating the derivation of adjoint and tangent linear models, conducting a generalized stability analysis is now straightforward, even for parallel discretizations of complex nonlinear coupled time-dependent problems. The widespread applicability of the framework was demonstrated in examples drawn from geophysical fluid dynamics, phase separation, and quantum mechanics.

Adjoint and tangent linear models arise across computational mathematics, not merely in stability analysis. Therefore, the same core technology of the automated derivation of adjoint and tangent linear models has major applications in optimization constrained by PDEs, automated error analysis and goal-based adaptivity, continuation and bifurcation analysis, data assimilation, and uncertainty quantification.

A further setting where adjoints prove very useful is Markov chain Monte Carlo (MCMC) algorithms that are used for Bayesian inference problems. It has been shown that if the derivative of the observation model is available, then the convergence of the algorithm is considerably faster [53, 43]. The derivative is also useful for avoiding getting stuck in local maxima [7]. Bayesian inverse problems have been recently rigorously formulated on function spaces in a well-posed manner; this means that MCMC algorithms can be appropriately modified so that the number of iterations required to converge is independent of mesh resolution [11]. The possibility of automated adjoint generation opens up the possibility of applying these algorithms in a very broad range of applications where they would not otherwise reach.

Another area of particular relevance to this work is the application of techniques from optimal control to transient growth and bypass transition: whereas generalized stability theory accounts for nonnormal effects, such analyses account for both nonnormal and nonlinear effects $[45,28,29]$. These techniques rely fundamentally on the solution of the associated adjoint system to provide the gradient information necessary for the nonlinear optimization. Future work will be to explore these applications and extend these techniques to physical systems where their implementation was previously impractical.

Acknowledgments. The secondary instability in salt fingers was originally discovered by F. P. MacTavish. The authors would like to thank G. N. Wells for providing the Cahn-Hilliard solver, and the anonymous reviewers for their thorough and constructive reviews. 


\section{REFERENCES}

[1] C. Abert, L. Exl, G. Selke, A. Drews, and T. Schrefl, Numerical methods for the strayfield calculation: A comparison of recently developed algorithms, J. Magnetism Magnetic Materials, 326 (2013), pp. 176-185.

[2] M. S. Alnæs, UFL: A finite element form language, in Automated Solution of Differential Equations by the Finite Element Method, A. Logg, K. A. Mardal, and G. N. Wells, eds., Springer, 2011, pp. 299-334.

[3] M. S. Alnes, A. Logg, K. B. Ølgandd, M. E. Rognes, and G. N. Wells, Unified Form Language: A Domain-Specific Language for Weak Formulations of Partial Differential Equations, ACM Trans. Math. Softw., to appear.

[4] S. Balay, J. Brown, K. Buschelman, V. Eijkhout, W. D. Gropp, D. Kaushik, M. G. Knepley, L. C. McInnes, B. F. Smith, and H. Zhang, PETSc Users Manual, Tech. Report ANL-95/11, Revision 3.2, Argonne National Laboratory, 2011.

[5] S. Balay, W. D. Gropp, L. C. McInnes, and B. F. Smith, Efficient management of parallelism in object oriented numerical software libraries, in Modern Software Tools in Scientific Computing, E. Arge, A. M. Bruaset, and H. P. Langtangen, eds., Birkhäuser, 1997, pp. 163-202.

[6] D. Barkley, H. M. Blackburn, and S. J. Sherwin, Direct optimal growth analysis for timesteppers, Internat. J. Numer. Methods Fluids, 57 (2008), pp. 1435-1458.

[7] A. Beskos, F. J. Pinski, J. M. Sanz-Serna, and A. M. Stuart, Hybrid Monte Carlo on Hilbert spaces, Stochastic Process. Appl., 121 (2011), pp. 2201-2230.

[8] S. N. Bose, Plancks Gesetz und Lichtquantenhypothese, Z. Phys., 26 (1924), pp. 178-181.

[9] J. W. CAhn And J. E. Hilliard, Free energy of a nonuniform system. I. Interfacial free energy, J. Chem. Phys., 28 (1958), pp. 258-267.

[10] I. Capuzzo Dolcetta, S. Finzi Vita, and R. March, Area-preserving curve-shortening flows: From phase separation to image processing, Interfaces Free Bound., 4 (2002), pp. 325-343.

[11] S. L. Cotter, M. Dashti, And A. M. Stuart, Approximation of Bayesian inverse problems for PDEs, SIAM J. Numer. Anal., 48 (2010), pp. 322-345.

[12] T. Davies, M. J. P. Cullen, A. J. Malcolm, M. H. Mawson, A. Staniforth, A. A. White, AND N. Wood, A new dynamical core for the Met Office's global and regional modelling of the atmosphere, Quart. J. Roy. Meteorological Soc., 131 (2005), pp. 1759-1782.

[13] J. M. Davis and S. M. Troian, Generalized linear stability of noninertial coating flows over topographical features, Phys. Fluids, 17 (2005), 072103.

[14] A. Einstein, Quantentheorie des einatomigen idealen Gases, Sitzungsberischte der Preussische Akademie der Wissenschaften, (1924).

[15] I. Eisenman, Non-normal effects on salt finger growth, J. Phys. Oceanography, 35 (2005), pp. 616-627.

[16] B. F. FARRELL, The initial growth of disturbances in a baroclinic flow, J. Atmospheric Sci., 39 (1982), pp. 1663-1686.

[17] B. F. FArrell, Transient growth of damped baroclinic waves, J. Atmospheric Sci., 42 (1985), pp. $2718-2727$.

[18] B. F. Farrell and P. J. Ioannou, Generalized stability theory. Part I: Autonomous operators, J. Atmospheric Sci., 53 (1996), pp. 2025-2040.

[19] B. F. Farrell and P. J. Ioannou, Generalized stability theory. Part II: Nonautonomous operators, J. Atmospheric Sci., 53 (1996), pp. 2041-2053.

[20] C. P. FArrell, Simulating Ultracold Matter: Horizons and Slow Light, Ph.D. thesis, University of St. Andrews, 2008.

[21] P. E. Farrell, D. A. Ham, S. W. Funke, and M. E. Rognes, Automated derivation of the adjoint of high-level transient finite element programs. SIAM J. Sci. Comput., 35 (2013), pp. C369-C393.

[22] M. Förster, U. Naumann, and J. Utke, Toward Adjoint OpenMP, Tech. Report AIB-201113, RWTH Aachen, 2011.

[23] A. Griewank and A. Walther, Evaluating Derivatives: Principles and Techniques of Algorithmic Differentiation, 2nd ed., SIAM, Philadelphia, 2008.

[24] E. Gross, Structure of a quantized vortex in boson systems, Il Nuovo Cimento, 20 (1961), pp. 454-477.

[25] J. Hake, A. G. Edwards, Z. Yu, P. M. Kekenes-Huskey, A. P. Michailova, J. A. McCammon, M. J. Holst, M. Hoshijima, And A. D. MCCulloch, Modelling cardiac calcium sparks in a three-dimensional reconstruction of a calcium release unit, J. Physiol., 590 (2012), pp. 4403-4422.

[26] V. Hernández, J. E. Román, A. Tomás, and V. Vidal, Krylov-Schur methods in SLEPc, 
Tech. Report STR-7, Universitat Politècnica de València, 2007.

[27] V. Hernandez, J. E. Roman, And V. Vidal, SLEPc: A scalable and flexible toolkit for the solution of eigenvalue problems, ACM Trans. Math. Software, 31 (2005), pp. 351-362.

[28] M. P. Juniper, Transient growth and triggering in the horizontal Rijke tube, Internat. J. Spray Combustion Dynam., 3 (2011), pp. 209-224.

[29] M. P. Juniper, Triggering in the horizontal Rijke tube: Non-normality, transient growth and bypass transition, J. Fluid Mech., 667 (2011), pp. 272-308.

[30] E. Kalnay, Atmospheric Modeling, Data Assimilation and Predictability, Cambridge University Press, Cambridge, UK, 2002.

[31] R. C. KIRBY, Algorithm 839: FIAT, a new paradigm for computing finite element basis functions, ACM Trans. Math. Software, 30 (2004), pp. 502-516.

[32] R. C. Kirby and A. LogG, A compiler for variational forms, ACM Trans. Math. Software, 32 (2006), pp. 417-444.

[33] O. Lahav, A. Itah, A. Blumkin, C. Gordon, S. Rinott, A. Zayats, and J. Steinhauer, Realization of a sonic black hole analog in a Bose-Einstein condensate, Phys. Rev. Lett., 105 (2010), 240401.

[34] C. LANCzos, An iteration method for the solution of the eigenvalue problem of linear differential and integral operators, J. Res. Natl. Bur. Standards, 45 (1950), pp. 255-282.

[35] R. LeIne, The historical development of classical stability concepts: Lagrange, Poisson and Lyapunov stability, Nonlinear Dynam., 59 (2010), pp. 173-182.

[36] A. LogG, K. A. Mardal, G. N. Wells, et Al., Automated Solution of Differential Equations by the Finite Element Method, Springer, 2011.

[37] A. Logg and G. N. Wells, DOLFIN: Automated finite element computing, ACM Trans. Math. Software, 37 (2010).

[38] E. N. Lorenz, A study of the predictability of a 28-variable atmospheric model, Tellus, 17 (1965), pp. 321-333.

[39] A. M. Lyapunov, The General Problem of the Stability of Motion, Control Theory and Applications Series, Taylor \& Francis, 1892. Translated by A. T. Fuller.

[40] F. P. MacTavish, The Application of Adaptive Mesh Techniques to Convective Processes in Oceanography, Ph.D. thesis, Imperial College London, Kensington, London, UK, 2013.

[41] X. Mao, S. Sherwin, And H. BlackBurn, Transient growth and bypass transition in stenotic flow with a physiological waveform, Theoret. Comput. Fluid Dynam., 25 (2011), pp. 31-42.

[42] G. R. Markall, A. Slemmer, D. A. Ham, P. H. J. Kelly, C. D. Cantwell, and S. J. SHERwin, Finite element assembly strategies on multi-core and many-core architectures, Internat. J. Numer. Methods Fluids, 71 (2012), pp. 80-97.

[43] J. Martin, L. C. Wilcox, C. Burstedde, and O. Ghattas, A stochastic Newton MCMC method for large-scale statistical inverse problems with application to seismic inversion, SIAM J. Sci. Comput., 34 (2012), pp. A1460-A1487.

[44] C. Moler And C. VAn LoAn, Nineteen dubious ways to compute the exponential of a matrix, twenty-five years later, SIAM Rev., 45 (2003), pp. 3-49.

[45] A. Monokrousos, A. Bottaro, L. Brandt, A. Di Vita, and D. S. Henningson, Nonequilibrium thermodynamics and the optimal path to turbulence in shear flows, Phys. Rev. Lett., 106 (2011), 134502.

[46] A. M. Moore, H. G. Arango, E. Di Lorenzo, B. D. Cornuelle, A. J. Miller, And D. J. NeILSON, A comprehensive ocean prediction and analysis system based on the tangent linear and adjoint of a regional ocean model, Ocean Modelling, 7 (2004), pp. 227-258.

[47] U. Naumann, The Art of Differentiating Computer Programs: An Introduction to Algorithmic Differentiation, Software Environ. Tools 24, SIAM, Philadelphia, 2012.

[48] K. B. ØlgaARd and G. N. Wells, Optimizations for quadrature representations of finite element tensors through automated code generation, ACM Trans. Math. Software, 37 (2010), pp. 8:1-8:23.

[49] T. M. ÖzGökmen and O. E. Esenkov, Asymmetric salt fingers induced by a nonlinear equation of state, Phys. Fluids, 10 (1998), pp. 1882-1890.

[50] T. N. Palmer, Medium and extended range predictability and stability of the Pacific/North American mode, Quart. J. Roy. Meteorological Soc., 114 (1988), pp. 691-713.

[51] P. C. PARKS, A. M. Lyapunov's stability theory -100 years on, IMA J. Math. Control Inform., 9 (1992), pp. 275-303.

[52] L. P. PitaevskiI, Vortex lines in an imperfect Bose gas, Soviet Phys. JETP, 13 (1961), pp. 451454.

[53] G. O. Roberts and R. L. Tweedie, Exponential convergence of Langevin distributions and their discrete approximations, Bernoulli, 2 (1996), pp. 341-363.

[54] M. E. Rognes And R. Winther, Mixed finite element methods for viscoelasticity with weak 
symmetry, Math. Models Methods Appl. Sci., 20 (2010), pp. 955-985.

[55] P. J. Schmid, Nonmodal stability theory, Annu. Rev. Fluid Mech., 39 (2007), pp. 129-162.

[56] M. E. Stern, The "salt-fountain" and thermohaline convection, Tellus, 12 (1960), pp. 172-175.

[57] G. W. Stewart, A Krylov-Schur algorithm for large eigenproblems, SIAM J. Matrix Anal. Appl., 23 (2001), pp. 601-614.

[58] L. N. Trefethen and D. BAu, Numerical Linear Algebra, SIAM, Philadelphia, 1997.

[59] L. N. Trefethen and M. Embree, Spectra and Pseudospectra: The Behavior of Nonnormal Matrices and Operators, Princeton University Press, Princeton, NJ, 2005.

[60] L. N. Trefethen, A. E. Trefethen, S. C. Reddy, and T. A. Driscoll, Hydrodynamic stability without eigenvalues, Science, 261 (1993), pp. 578-584.

[61] S. Tremaine, On the origin of irregular structure in Saturn's rings, Astronom. J., 125 (2003), p. 894 .

[62] J. S. Turner, Multicomponent convection, Annu. Rev. Fluid Mech., 17 (1985), pp. 11-44.

[63] W. G. Unruh, Experimental black-hole evaporation?, Phys. Rev. Lett., 46 (1981), pp. 13511353.

[64] J. Utke, L. Hascoet, P. Heimbach, C. Hill, P. Hovland, and U. Naumann, Toward adjoinable MPI, in Proceedings of the IEEE International Symposium on Parallel Distributed Processing (IPDPS 2009), Rome, Italy, 2009, pp. 1-8.

[65] L. Vynnytska, M. E. Rognes, and S. R. Clark, Benchmarking FEniCS for mantle convection simulations, Comput. Geosci., 50 (2013), pp. 95-105.

[66] G. N. Wells, E. Kuhl, and K. Garikipati, A discontinuous Galerkin method for the CahnHilliard equation, J. Comput. Phys., 218 (2006), pp. 860-877.

[67] K. Wu And H. Simon, Thick-restart Lanczos method for large symmetric eigenvalue problems, SIAM J. Matrix Anal. Appl., 22 (2000), pp. 602-616.

[68] L. Zanna, P. Heimbach, A. M. Moore, and E. Tziperman, Optimal excitation of interannual Atlantic meridional overturning circulation variability, J. Climate, 24 (2011), pp. 413-427.

[69] L. Zanna, P. Heimbach, A. M. Moore, and E. Tziperman, Upper-ocean singular vectors of the North Atlantic climate with implications for linear predictability and variability, Quart. J. Roy. Meteorological Soc., 138 (2012), pp. 500-513. 